\title{
Aerial Social Force Model: A New Framework to Accompany People Using Autonomous Flying Robots
}

\author{
A. Garrell ${ }^{1 *}$, Luis Garza-Elizondo ${ }^{1 *}$, M. Villamizar ${ }^{2}$, F. Herrero ${ }^{1}$ and A. Sanfeliu ${ }^{1}$ \\ *indicates equal contributions
}

\begin{abstract}
In this paper, we propose a novel Aerial Social Force Model (ASFM) that allows autonomous flying robots to accompany humans in urban environments in a safe and comfortable manner. To date, we are not aware of other stateof-the-art method that accomplish this task. The proposed approach is a 3D version of the Social Force Model (SFM) for the field of aerial robots which includes an interactive human-robot navigation scheme capable of predicting human motions and intentions so as to safely accompany them to their final destination. ASFM also introduces a new metric to finetune the parameters of the force model, and to evaluate the performance of the aerial robot companion based on comfort and distance between the robot and humans. The presented approach is extensively validated in diverse simulations and real experiments, and compared against other similar works in the literature. ASFM attains remarkable results and shows that it is a valuable framework for social robotics applications, such as guiding people or human-robot interaction.
\end{abstract}

\section{INTRODUCTION}

In recent years, we have seen extensive progress in the field of robotics and machine learning. At present, ground and aerial robots can be found for a wide range of applications, such as transportation, entertainment, and domestic tasks. Despite this progress, robot navigation is one of the most difficult challenges which researchers face, mainly because real environments present obstacles and highly dynamic objects moving in different directions and velocities, making it difficult to accomplish safe navigation towards a final destination.

Research on aerial robots has also grown substantially, thanks to modern advances in technology, materials, and systems of perception and control. This research paves the way for a near future wherein aerial and ground robots interact with people in houses, streets, or retail environments in order to perform specific tasks.

To accomplish this goal, the interaction between robots and humans plays a key role, since robots must learn and act in accordance with human behaviors and observe certain

\footnotetext{
${ }^{1}$ The authors are with Institut de Robotica i Informatica Industrial (CSICUPC), Barcelona, Spain, \{agarrell, lgarza, fherrero, sanfeliu\}eiri.upc.edu

2 The author is with Idiap Research Institute, Martigny, Switzerland michael.villamizareidiap.ch

This work is supported by the Spanish State Research Agency through the Mara de Maeztu Seal of Excellence to IRI MDM-2016-0656.

This work was supported by the Spanish Ministry of Science and Innovation, project ColRobTransp DPI2016-78957-R (AEI/FEDER, UE) and AEROARMS European project H2020-ICT-2014-1-644271.
}

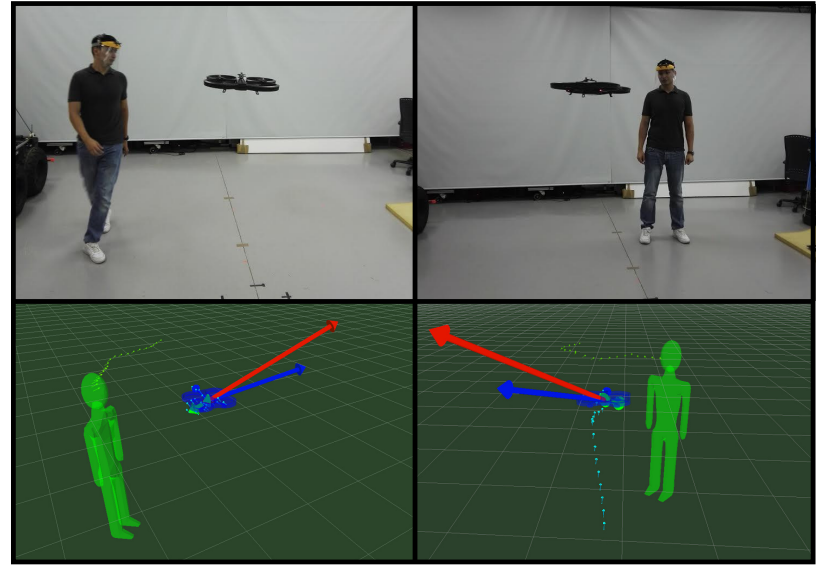

Fig. 1: Aerial robot accompanying a person. ASFM computes the interactive forces which enable the robot navigate side-by-side with a human. Top row: Two instances of a person interplaying with the robot. Bottom row: The same scenes depicting the aerial social forces provided by ASFM.

social conventions, such as maintaining the level of personal space by which people feel safe and comfortable.

Nowadays, this is a subject of study where efforts are mainly focused on the development of autonomous companion robots capable of performing human-robot interactions in a more natural way [1], [2]. However, these works were developed for ground robots using 2D social force models. Similarly, other works for robot companion introduce mediating factors, such as person's individual experience with robots [3] or gender identity [4], albeit, the experiments were conducted on controlled areas with no dynamic obstacles nor people.

With regards to aerial robots, the research on robot companion is relatively minimal [5], [6].Furthermore, the works have been mainly devoted to simple robotics tasks with little interaction between the robot and the person.

Unlike previous works for ground robots, we propose a new robot companion approach for aerial robots in urban environments. This approach, called Aerial Social Force Model (ASFM), defines a social interaction model between persons, robots, and objects (refer to Fig. 1). Particularly, ASFM is an 3D extension of the Social Force Model (SFM), introduced by Helbing [7], in order to consider the possibility to navigate in real world in different altitudes instead of moving in a plane. 
The proposed approach consists of three main stages. In the first stage, ASFM includes an efficient and accurate prediction module based on online linear regression to estimate the person's trajectory [8]. This module allows the flying robot to accompany the person by his or her side in order to provide a higher degree of comfort and safety for the person.

This contrasts with other methods based on following people without inferring the intention of people. The output of this module is a set of future person's positions, whereas the input are the current positions of the person and aerial robot acquired by the Optitrack motion capture system used in our experiments.

The second stage of ASFM corresponds to the computation of the interacting forces that determine the behavior of the robot during the accompaniment. To apply the required forces, we learned the appropriate parameters of the social force model. This is carried out in two steps. Initially, the force parameters are computed using a genetic algorithm [9] and a database created for this goal during a training phase. In the second step, we adjust the model's parameters using post-experiment questionnaires to get human feedback about the robot's behavior and the person comfort during the companion task.

Finally, in the last stage, we introduce a new quantitative metric to evaluate the robot's performance. Since the verification of man-in-the-loop systems is fuzzy, we developed an quantitative metric that evaluates the behavior of our robot companion approach, during the task the robot tries to maximize this performance.

The remainder of the paper is organized as follows. Section II describes the Aerial Social Force Model (ASFM) and its main components. In Section III, ASFM is evaluated exhaustively in diverse simulations and real-life experiments. Finally, Section IV provides the conclusions.

\section{Aerial Social Force Model}

In this section, we proceed to describe the different modules of the proposed ASFM approach: The regression model used to estimate people's motion, the interactive forces of the aerial force model, and the quantitative metric used to evaluate the performance of the robot.

\section{A. Online Regression Model}

In this work, we make use of the well-known regression model to estimate people's future positions in order to let the robot navigate side-by-side with humans. The coordinates of the person and the aerial robot positions are acquired using the Optitrack sensing system, including an array with the differential values between consecutive values. Subsequently, the coefficients of the model are computed using Stochastic Gradient descent (SGD) [8]. This process is online, and therefore, the robot computes continuously the estimation of people motion and uses this information to calculate the interactive social forces.

$$
f(X)=w_{0}+\sum_{j=1}^{p} X_{j} w_{j}
$$

Where the input vector is defined as $X=$ $\left(\Delta x_{1}, \Delta x_{2}, \ldots, \Delta x_{p}\right)$ and $\mathbf{w}=\left(w_{1}, \ldots, w_{p}\right)$ are the unknown coefficients to be adjust, to finally predict a real-valued output Y.

To estimate the coefficients, $X$ and $Y$ matrices are built, the number of previous values to take into account in this case are five, this means, six unknown parameters need to adjust. During the optimization process the gradient descent method was used, and the number of examples $N$ was one hundred.

$$
\begin{gathered}
X=\left[\begin{array}{ccccc}
1 & \Delta x_{n_{1}, 1} & \Delta x_{n_{2}, 2} & \cdots & \Delta x_{n_{p}, p} \\
1 & \Delta x_{n_{2}, 1} & \Delta x_{n_{3}, 2} & \cdots & \Delta x_{n_{p+1}, p} \\
\vdots & \vdots & \vdots & \ddots & \vdots \\
1 & \Delta x_{n_{N-p}, 1} & \Delta x_{n_{N-p+1}, 2} & \cdots & \Delta x_{n_{N-1}, p}
\end{array}\right] \\
y=\left[\Delta x_{p+1}, \Delta x_{p+2}, \ldots, \Delta x_{N}\right]^{\prime}
\end{gathered}
$$

With the previously collected data, and the matrices built, the weights were found using the well-known method gradient descent optimization method, with an initial random guess of the parameters. The cost function was defined as:

$$
J(\mathbf{w})=\sum_{i=1}^{N}(f(X)-y)^{2}
$$

This method works in an iterative way, in which in each iteration the weights are updated in order to be closer to the optimal minimum, changing the weights in each iteration $k$ in the direction of the gradient, seeking that:

$$
J\left(w_{0}^{k+1}, w_{1}^{k+1}, \ldots, w_{p}^{k+1}\right)<J\left(w_{0}^{k}, w_{1}^{k}, \ldots, w_{p}^{k}\right)
$$

Until the cost function is not reduced anymore, or until the iteration cycle finishes. The gradient of the cost function is defined as follows:

$$
\begin{gathered}
\nabla J(\mathbf{w})=\left(\frac{\partial J}{\partial w_{0}}, \frac{\partial J}{\partial w_{1}}, \ldots, \frac{\partial J}{\partial w_{p}}\right) \\
\frac{\partial J}{\partial w_{p}}=\frac{1}{N} \sum_{i=1}^{N}\left(\sum_{j=0}^{p} X_{i j} w_{j}-y_{i}\right) X_{p}
\end{gathered}
$$

In order to move through the gradient, the RMSprop strategy is used, this method was proposed by Geoff Hinton in an unpublished work, and it states that dividing the gradient by the root of the expected value of the gradient, taking into account a short window, makes the learning work much better.

$$
E\left[\nabla J(\mathbf{w})^{2}\right]_{t}=\gamma E\left[\nabla J(\mathbf{w})^{2}\right]_{t-1}+(1-\gamma) \nabla J(\mathbf{w})_{t}^{2}
$$

where $\gamma$ means the momentum value, typically set to 0.9 or 0.95 , and always $<1$.

$$
\Delta w_{t}=-\eta \cdot \nabla J(\mathbf{w})
$$

In which $\eta$ means the learning rate set by the user.

$$
w_{t+1}=w_{t}+\Delta w_{t}
$$




$$
w_{t+1}=w_{t}-\frac{\eta}{\sqrt[2]{E\left[\nabla J(\mathbf{w})^{2}\right]_{t}+\epsilon}} \nabla J(\mathbf{w})
$$

where $E\left[\nabla J(\mathbf{w})^{2}\right]_{t}$ means the decaying average over past squared gradients, and $\epsilon$ is a smoothing term that avoid the division by zero value.

\section{B. Interactive Forces}

In this section, we proceed to describe a model capable of representing the interactions between a pedestrian and a flying robot, based on the works of [7], [10]. Their researches state that changes in behavior (trajectory) can be explained in terms of social fields or forces. Nevertheless, in these works their representation models are in 2-dimensions, while in the present paper, we introduce an extension of these models adding a new dimension. Also, they do not take into account the interaction between people and aerial robots, which are two of the main contributions of the this work.

In the social force model, pedestrian interactions and final destinations are defined as a summation of existing interactive forces that determines the resultant force, and hence, people's trajectories. The model states the final motion which can be expressed through a function depending on the pedestrians' positions and velocities. In the Aerial Social Force Model (ASFM), we introduce a flying robot in the social environment, and take into account the interaction among the robot, people and obstacles.

More precisely, the total force is defined by the sum of the robot-humans and robot-objects interaction forces, and the attraction forces, that makes the robot being closer to the human being accompanied,

$$
\mathbf{F}_{\mathbf{R}}=\mathbf{f}_{\mathbf{R}}^{\text {goal }}+\mathbf{F}_{\mathbf{R}}^{\text {int }}
$$

The interaction force $\mathbf{F}_{\mathbf{R}}^{\text {int }}$ is the summation of all the repulsive forces exerted by other pedestrians, objects and robot around him, and is defined as:

$$
\mathbf{F}_{\mathbf{R}}^{\mathbf{i n t}}=\sum_{p j \in P} \mathbf{f}_{\mathbf{R j}}^{\mathbf{i n t}}+\sum_{o \in O} \mathbf{f}_{\mathbf{R o}}^{\text {int }}
$$

where $P$ is the set of people moving in the environment and $O$ is the set of obstacles. Next, it is defined each interaction force affecting the robot individually, and the motivation behind this.

1) Goal force attraction: This force drives the robot to the goal. In our case, there are two attraction forces, one which pursues the position of the human being accompanied, and the second one that pushes the robot to the human forecasted position in the next future steps. The robot will adapt its actual velocity $v_{R}$ with a certain relaxation time $k^{-1}$. Both attraction forces are defined as:

$$
\mathbf{f}_{\mathbf{R}}^{\text {goal }}=k\left(\mathbf{v}_{\mathbf{R}}^{\mathbf{0}}-\mathbf{v}_{\mathbf{R}}\right)
$$

Formally, the social forces model assumes that a robot $R$ with mass $\mathrm{m}_{i}$ tries to move at a certain desired speed $v_{R}^{0}$ in a desired direction $\mathbf{e}_{R}$, i.e., with desired velocity $\mathbf{v}_{\mathbf{R}}^{\mathbf{0}}=v_{R}^{0} e_{R}$.
2) Robot-human interaction force: Pedestrians also exert a repulsive force over the robot. In the same way, people want to keep a certain distance to the robots and other pedestrians and objects, because whether they are too close to the robot, they might feel uncomfortable. One of the main contributions of this work is the extension of the study of the pedestrian's and robot's"personal" space, which in the previous work [1] was well studied taking into account a land-based robot interplaying with pedestrians,

$$
\mathbf{f}_{\mathbf{R} \mathbf{j}}^{\mathbf{i n t}}=A_{R j} e^{\left(\frac{d_{R}-d_{R j}}{B_{R j}}\right)} \frac{\mathbf{r}_{\mathbf{R j}}(\mathbf{t})}{d_{R j}(t)} \psi\left(\varphi_{R j}, \theta_{R j}\right)
$$

where $A_{R j}, B_{R j}, \lambda_{R j}, d_{R}$ are the parameters to be learned. $\psi\left(\varphi_{R j}, \theta_{R j}\right)$ represents the anisotropic factor in a 3D space, which depends on $\varphi_{R j}$, an angle formed between the desired velocity of the pedestrian $p_{i}$ and the vector $\mathbf{r}_{\mathbf{R j}}$ (which is the distance between the robot and the pedestrian $p_{j}$ and pointing to him) and $\theta_{R j}$, which is the angle between the $x$ and $z$ coordinates, an angle formed between the position of the robot and the pedestrian. $\psi\left(\varphi_{R j}, \theta_{R j}\right)$ is defined as:

$$
\psi\left(\varphi_{R j}, \theta_{R j}\right)=w\left(\varphi_{R j}\right) \cos \left(\theta_{R j}\right)\left(h+\xi_{R j} w\left(\varphi_{R j}\right)\right)
$$

where $h$ is the height of the pedestrian, Further, $w\left(\varphi_{R j}\right)$ is described as:

$$
w\left(\varphi_{R j}\right)=\lambda_{R j}+\left(1-\lambda_{R j}\right)\left(\frac{1+\cos \left(\varphi_{R j}\right)}{2}\right)
$$

where $\lambda_{R j}$ defines the strength of the anisotropic, and $\cos \left(\varphi_{i R}\right)$ is calculated as:

$$
\cos \left(\varphi_{R j}\right)=-\mathbf{n}_{\mathbf{R j}} \cdot \mathbf{e}_{\mathbf{p j}}
$$

$\mathbf{n}_{\mathbf{R j}}$, which describes the direction of the force, is the normalized vector pointing from the robot to $p_{j}$ and $\mathbf{e}_{\mathbf{p j}}$ is the desired motion direction of the pedestrian $p_{j}$ (which is pointing to the goal).

The robot anisotropic factor has some differences with respect to the anosotropic factor of the humans, due that the drone is on the air all the time and does not has the same limitations as in the ground. This new anisotropic factor is defined by:

$$
\psi\left(\varphi_{R j}, \theta_{R j}\right)=w\left(\varphi_{R j}\right) \cos \left(\theta_{R j}\right)
$$

In Fig. 2, it is shown the representations of the anisotropic factors (personal spaces) of both people and flying robots. In order to determine these values, we conducted a series of tests in which a flying robot moved towards a person at different speeds $(0.4 \mathrm{~m} / \mathrm{s}, 0.7 \mathrm{~m} / \mathrm{s}$ and $0.9 \mathrm{~m} / \mathrm{s})$, at different angles $\left(0^{\circ}, 45^{\circ}, 90^{\circ}, 135^{\circ}\right.$ and $\left.180^{\circ}\right)$ and height $(0.5 \mathrm{~m}, 0.75 \mathrm{~m}$, $1 \mathrm{~m}, 1.25 \mathrm{~m}, 1.5 \mathrm{~m}, 1.75 \mathrm{~m}, 2 \mathrm{~m})$. 


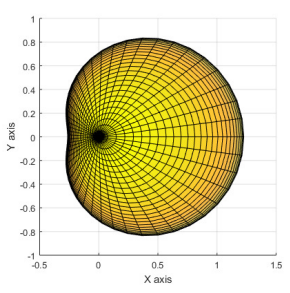

(a)

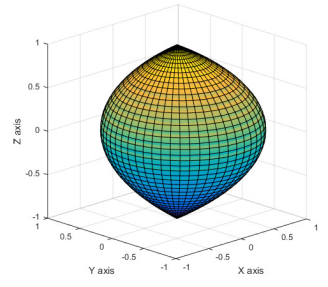

(e)

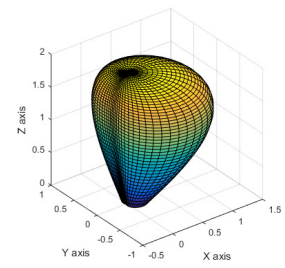

(b)

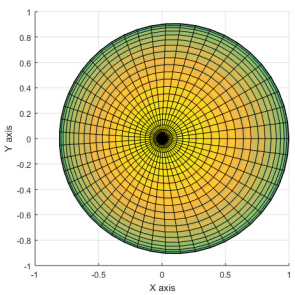

(f)

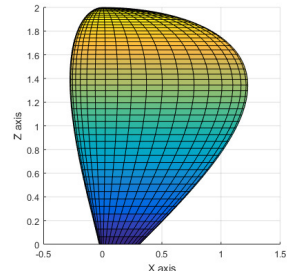

(c)

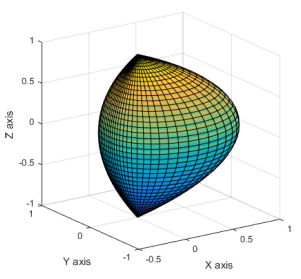

$(\mathrm{g})$

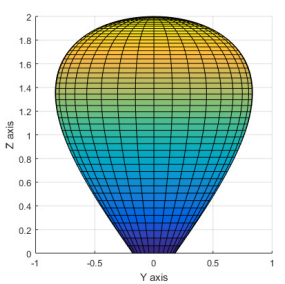

(d)

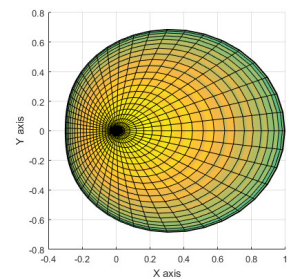

(h)

Fig. 2: Anisotropic factor in 3D view . Top row: Human's anisotropic factor in 3D view , with $\lambda_{R j}=0.25$ and $\xi_{R j}=0.25$. Bottom row: Drone's anisotropic factor in 3D view, (e) and (f) with $\lambda_{1}=0.90$ and (g) and (h) with $\lambda=0.50$

3) Robot-object interaction force: The flying robot, as well as humans do, keeps a certain distance from objects when navigates, trying to follow an objects-free path to safely reach its goal. Therefore, the objects around the flying robot exert a force diverting its path, with a repulsive and monotonic decreasing potential $U_{i o}$ [7]. This repulsive force is defined as:

$$
\mathbf{f}_{\mathbf{R o}}^{\text {int }}=-\nabla_{r_{R o}} U_{R o}\left(\left\|\mathbf{r}_{\mathbf{R o}}\right\|\right)
$$

where $U_{R o}\left(\left\|r_{R o}\right\|\right)$ is defined as:

$$
U_{R o}\left(\left\|\mathbf{r}_{\mathbf{R o}}\right\|\right)=U_{R o}^{0} e^{\frac{-\left\|\mathbf{r}_{\mathbf{R o}}\right\|}{C}},
$$

the vector $\mathbf{r}_{\mathbf{R o}}$ is defined as the difference between the location of the robot and the location of the obstacle $o$ that is nearest to the robot.

The total force which will drive the flying robot is defined by:

$$
F_{R}=f_{R, \text { dest }}^{\text {goal }}+f_{R j}^{\text {goal }}+F_{R j}^{\text {int }}+F_{R o}^{\text {int }}
$$

Constraints in drone's velocity has also be taken into account, depending on how close the aerial robot is. We have defined zones inspired on how people interacts and in function of the pedrestrian proximity:

$$
v_{R}= \begin{cases}v_{\text {safety }} & \frac{d_{R j}}{w\left(\varphi_{R j}\right)} \leq \mu_{\text {safety }} \\ v_{\text {cruise }} & \mu_{\text {safety }}<\frac{d_{R j}}{w\left(\varphi_{R j}\right)} \leq \mu_{\text {social }} \\ v_{\text {free }} & \text { otherwise }\end{cases}
$$

being $v_{\text {safety }}$ the maximum velocity the drone can take, when is at least one person inside the inner safety zone. $v_{\text {cruise }}$ is the velocity when someone is inside its social safety zone and $v_{\text {free }}$ is the maximum velocity when no one is inside its safety zone.

\section{Quantitative Metrics}

To evaluate the performance of the task accomplished by the flying robot, a new quantitative metric is defined. This assessment is based on "proxemics", proposed by [11] and the importance to walk side-by-side. This work considers the following distances between people:

- Intimate distance: the presence of another person is unmistakable $(0-45 \mathrm{~cm})$.

- Personal distance: comfortable spacing $(45 \mathrm{~cm}-1.22 \mathrm{~m})$.

- Social distance: limited involvement (1.22m-3m).

- Public distance: outside circle of involvement $(>3 \mathrm{~m})$.

To define the metric used in the present work, four different areas have been defined: (i) People's Personal space $\mathcal{C}_{i}$, where the robot must not perturb the human's vital space, eq. 24. (ii) Social distance area $\mathcal{A}$, which the robots must respect to be socially accepted. (iii) The robot should be in the human's field of view to allow human-robot interaction during the performance of the task and must navigate sideby-side with the person $\mathcal{B}$. (iv) Finally, if there are other pedestrians in the environment $p_{j}$, the robot must not perturb pedestrians' personal space $\bigcup \mathcal{C}_{j}$.

$$
\begin{aligned}
\mathcal{A} & =\left\{x \in \mathbb{R}^{3} \backslash\left(\mathcal{B} \cup^{p_{j}} \mathcal{C}\right) \mid d\left(x, p_{i}\right)<3\right\} \\
\mathcal{B} & =\left\{x \in \mathbb{R}^{3} \backslash \mathcal{C} \mid d\left(x, p_{i}\right)<3 \psi\left(\varphi_{p_{i}}, \theta_{p_{i}}\right)\right\} \\
\mathcal{C} & =\left\{x \in \mathbb{R}^{3} \mid d\left(x, p_{i}\right)<\psi\left(\varphi_{p_{i}}, \theta_{p_{i}}\right)\right\}
\end{aligned}
$$

where $\psi\left(\varphi_{p_{i}}, \theta_{p_{i}}\right)$ is defined in eq. 16. Moreover, the flying robot is represented as $\psi\left(\varphi_{R j}, \theta_{R j}\right)$, previously introduced in eq. 19.

Thus, we can now define the performance of the task accomplished by the robot, depending on human's position $p_{i}$ and robot's position $p_{R}$.

$$
\rho\left(r, p_{i}\right)=\int_{\left(\mathcal{B} \backslash \bigcup_{p_{j}} \mathcal{C}_{j}\right) \cap \mathcal{R}} \frac{d \mathbf{x}}{|\mathcal{R}|}+\int_{\left(\mathcal{A} \backslash \bigcup_{p_{j}} \mathcal{C}_{j}\right) \cap \mathcal{R}} \frac{d \mathbf{x}}{2|\mathcal{R}|} \in[0,1]
$$




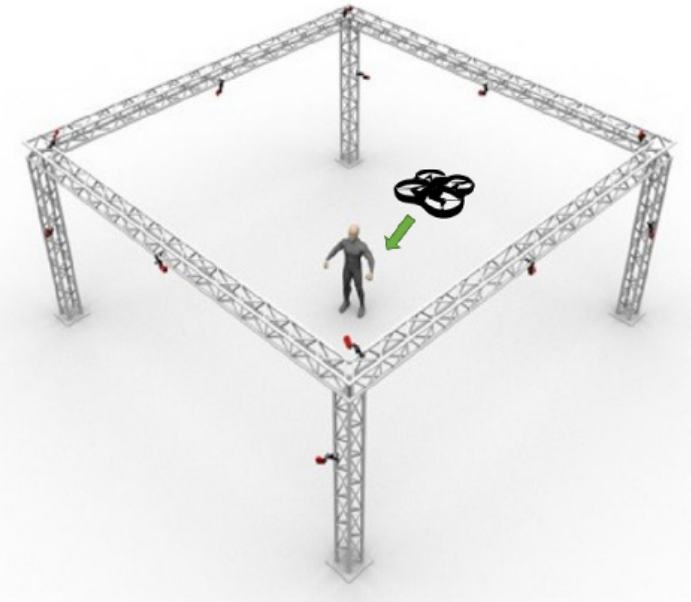

Fig. 3: Optitrack System. An indoor environment with Optitrack depicting the experiments we performed.

Where $\mathbf{x} \in \mathbb{R}^{3}$. The range of the performance function is defined between 0 and 1 . If the complete area of the robot is allocated in zone $\mathcal{B}$, the performance gets the maximum value, i.e., 1. As the robot moves far from the person and enters to zone $\mathcal{A}$, the performance decreases to 0.5 . Finally, the performance in zones $\mathcal{C}_{i}$ is 0 , as it is not allowed that the robot enters in people's personal space.

\section{EXPERIMENTS}

In the present section, we proceed to describe the synthetic and real-live experiments the autonomous flying robot has been performed according to the ASFM.

\section{A. Robotic Platform and Environment}

During the experiments, we have used the AR.Drone 2.0, a flying quadcopter build by Parrot. The quadcopter is a very good choice to test our algorithms in a short-time setup.

Moreover, we make use of the Optitrack Motion Capture system, it is a system created by NaturalPoint Inc. In this work, Optitrack was used to analyze an indoor environment to track the human and the drone's positions in a previously defined space. Optitrack allows us to calculate the interaction forces between the person and the robot and to see the behavior of our model in real time. Both, human and drone used markers in the body to let the software detect the exactly position of each of them. The working area for the experiments were $5 x 5 \mathrm{~m}$.

\section{B. Synthetic Experiments}

Here, we present the evaluation of the performance of the ASFM, for this reason, we have built a simulated social environment. In this section, we introduce the computation of the parameters that have been used in the model, the results of the implemented regression model to estimate person's motion and the simulations of the complete system.
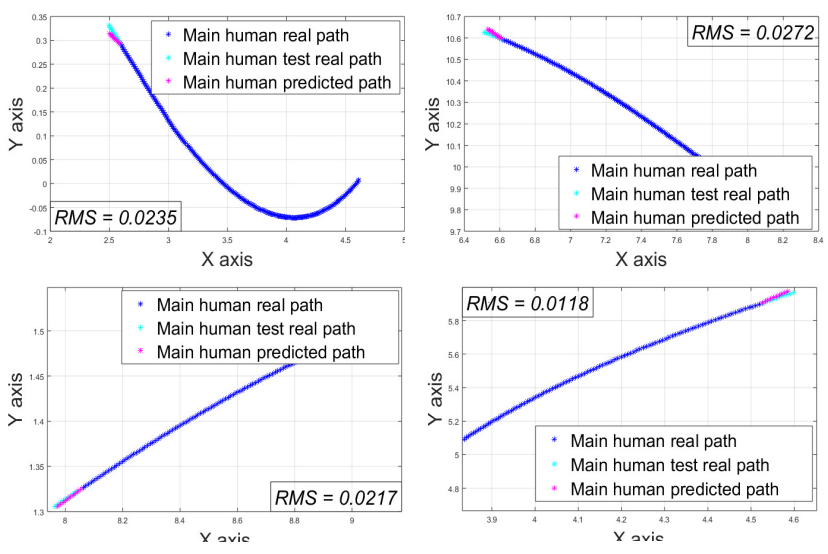

Fig. 4: Regression Model. Different humans' trajectories and the forecasted paths versus the real path. The error was measure using the Root-mean-square error.

1) Online Regression Model: During the synthetic experiments we simulated the social behavior of a set of pedestrians, the drone and the human being accompanied. Then, we collected the data, which help us to see an approximate real behavior of pedestrians walking along a path, and we used this data to build a model to forecast the next position few seconds ahead. The model is based on the differential values in $x$ and $y$ positions.

Different trajectories were tested with the regression model, always taking into account the last five human's positions, and then the path that the person will follow was built. One of the strongest points is the high accuracy prediction when the path is not too complicate, nevertheless, also good results were obtained when the path drastically changed as is shown in Fig. 4, first plot. With these results, the robot can move along the direction, navigating side-byside with the human being accompanied.

The drone's prediction capacity proved to be useful in highly dynamic environments, in which a lot of people walked around the accompanied human and the flying robot. In this situation, the walking behavior of the accompanied human can change unexpectedly, nevertheless the drone proves to adapt to the accompanied human with very good accuracy.

2) ASFM parameters: As described above, we consider three kinds of interaction forces: human-human, humanobstacle and human-drone. The two first interactions have been presented previously in [12], [13]. Moreover, in [10] a description of the interaction between a ground robot and a human is described. However, the human-drone interaction parameters were not obtained in any previous work, thereby, in this section we introduce the results obtained for the parameters $\left\{A_{R j}, B_{R j}, \lambda_{R j}, d_{R j}\right\}$.

We optimized the parameters of the force interaction model under the presence of a moving flying robot, where this interaction force is the only external force altering the outcome of the described trajectory. All optimizations used to learn the model forces parameters were carried out using 


\begin{tabular}{|l||c|c|c|c|c|}
\hline Interaction & $\mathrm{k}$ & $\mathrm{A}$ & $\mathrm{B}$ & $\mathrm{d}$ & $\lambda$ \\
\hline \hline Human-Human [13] & 2 & 1.25 & 0.1 & 0.2 & 0.5 \\
\hline Human-Human [12] & 4.9 & 10 & 0.34 & 0.16 & 1 \\
\hline Human-Robot [10] & 2.3 & 2.66 & 0.79 & 0.4 & 0.59 \\
\hline Human-Flying robot & 4.45 & 3.35 & 0.565 & 0.295 & 0.55 \\
\hline
\end{tabular}

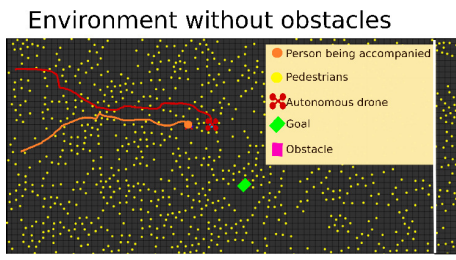

Environment with obstacles

TABLE I: Aerial Social Force Model Parameters. Comparison of the learned parameters in diverse works of humanhuman, human-robot and human-drone interactions after applying the minimization process.

genetic optimization algorithms [9], which minimize the following error function throughout all $\mathrm{N}$ training trajectories,

$$
\{A, B, \lambda, d\}=\arg \min _{\{A, B, \lambda, d\}}\left\{\sum_{N} \sum_{\text {time }}\left\|\mathbf{x}_{\mathbf{0}}(\mathbf{t})-\mathbf{x}_{\mathbf{e}}(\mathbf{t})\right\|\right\}
$$
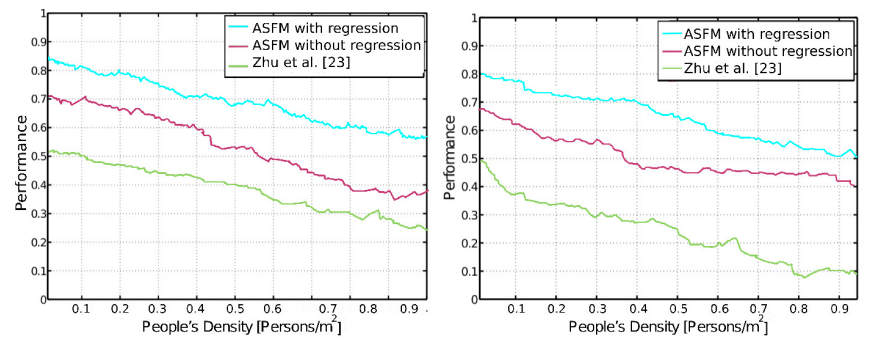

Table I shows the parameters learned after applying the minimization process in the set of recorded human-drone navigation trajectories and compared with the parameters obtained in other reserach works.

3) Simulations: To this end, we have implemented a complete social environment, depicted in the first row in Fig. 5, which takes into account pedestrians, obstacles and drones, where each element is reactive to its surrounding according to the ASFM. By doing this, we can get a dynamical environment, in which each action of the autonomous drone alters the behavior of the other pedestrians in the environment.

To evaluate mathematically the correctness and the performance of the presented model, we built a simulated social environment. This simulated environment allows us to validate the performance of the method, using the metrics defined in Sec. II-C, in different environments and under different pedestrian density.

In order to give statistical consistency to our results, more than $10 \mathrm{k}$ experiments have been carried out, only varying the initial position of each pedestrian in the simulation. We would like to emphasize on the fact that the environment has a high density of persons and each person aims to a random destination.

The bottom row of Fig. 5 shows the overall performance of the different methods with respect to the pedestrian density in the scene. It can be seen, that using our ASFM method, the performance highly increases. The predictive behavior using the regression model clearly enhances the performance of the task, in both scenarios.

\section{Real-live Experiments}

In this section, we present different real-live experiments, in which we tested the ASFM model, considering both, humans and obstacles. Two scenarios were tested, in the first one there are no obstacles, while in the second one there are different obstacles. The data was collected using the Optitrack system and we used a ROS visualization tool to show the position of the human and the drone, and the interaction forces.

During real-live experiments, we tested our model and its capacity to maintain the drone close to the human and

Fig. 5: Synthetic experiments. Top row: Simulated environments. Bottom row: Performance presented previously; blue line represents the ASFM with the regression model; purple refers to ASFM without regression, and green color is the performance of the work presented in [14]. All results are function of the pedestrian density in the environment.

accompany him/her in a comfortable manner. We noticed that all real experiments carried out with our robot and different volunteers achieved the goal, that is, the drone was able to accompany the human while navigating in a social and acceptable manner.

In Fig. 6 are shown three different scenarios of experiment trials. In all the scenarios, the top view is the picture of the trial and bottom view is the drawn of the interaction forces.

In the first scenario, two rows of images in the top of the figure, Fig. 6-top shows how a human walks around the robot in a closer way, and the bottom shows the repulsion forces drawn as red arrow that makes the robot move backward, respecting human's personal space.

In the second scenario, two rows of images in the middle of the figure, Fig. 6-middle shows how a human walks in the drone's direction as if they were to collide, and then the human moves backward quickly. In this case, the highest repulsion force is obtained when the distance between the drone and the human is minimum.

Finally, in the third scenario, two rows of images in the bottom of the figure, in Fig. 6-bottom we put a cylindrical obstacle between the drone and the human. The repulsion force that is exerting the object keeps the drone away, as long as the human is behind the object.

We would like to point the reader to check all the videos of synthetic and real experiments on following link http://www.iri.upc.edu/people/agarrell/ iros 2017 . html

\section{CONCLUSIONS}

This paper has presented a novel approach based on the Social Force Model. The major contributions of this chapter are twofold. On the one hand, we present a new Aerial Social Force model to accompany a human, and we explain how 

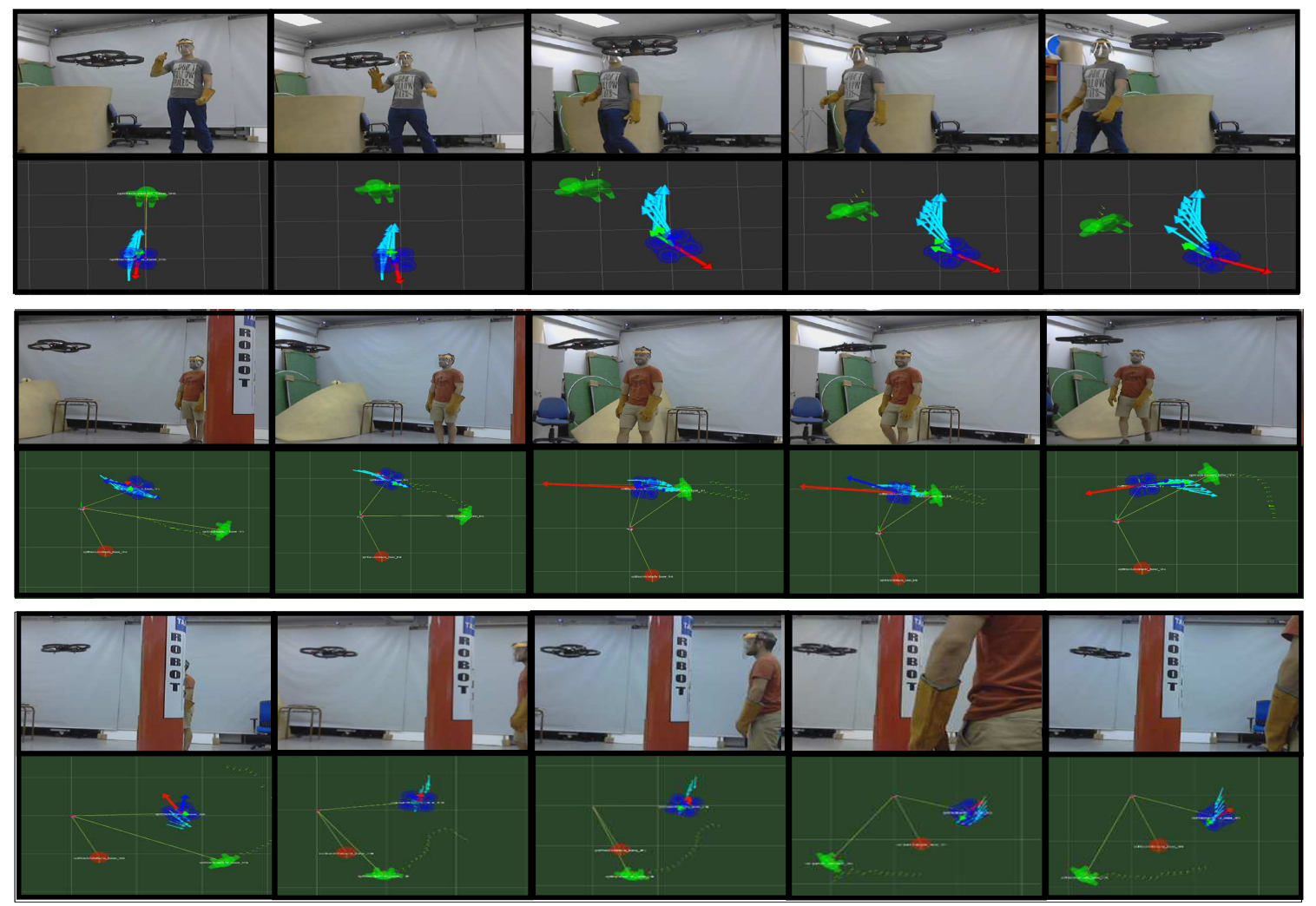

Fig. 6: Real-live experiments. Top row: Human moving through a certain path surrounding the drone, the drone moved backward. It is plotted the visualization of the scenario and representation of the interaction forces in real-time too. Middle row: Human moving forward in the direction of the drone, in the image below, the repulsion force depicted as a red arrow. Bottom row: Human moving around an obstacle, the drone stays in the same position as the sum of the forces was zero.

to obtain the force parameters of human-robot interactions specifically suited to the AR Drone.

On the other hand, we present a new evaluation metric. Since the verification of any system involving human participation is hard to evaluate objectively, we define an analytical metric that allows to measure the behavior of our autonomous flying robot when accompany a human.

We validated the model through an extensive set of simulations and real-live experiments. In contrast to other existing approaches, our method can handle realistic and dynamic situations, such as dealing with large environments littered with obstacles and dense crowds. For that reason, this work can be applied to various specific real-live robot applications; for instance, guiding tourists through crowded city streets.

In future work, we aim to use a visual system to detect humans' positions and thus, to work in outdoor environments without the necessity of relying on the Optitrack system.

\section{REFERENCES}

[1] A. Garrell and A. Sanfeliu, "Cooperative social robots to accompany groups of people," The International Journal of Robotics Research, vol. 31, no. 13, pp. 1675-1701, 2012.

[2] H. Ishiguro, T. Ono, M. Imai, T. Maeda, T. Kanda, and R. Nakatsu, "Robovie: an interactive humanoid robot," Industrial robot: An international journal, vol. 28, no. 6, pp. 498-504, 2001.

[3] K. L. Koay, E. A. Sisbot, D. S. Syrdal, M. L. Walters, K. Dautenhahn, and R. Alami, "Exploratory study of a robot approaching a person in the context of handing over an object." in AAAI spring symposium: multidisciplinary collaboration for socially assistive robotics, 2007, pp. 18-24.
[4] K. Dautenhahn, M. Walters, S. Woods, K. L. Koay, C. L. Nehaniv, A. Sisbot, R. Alami, and T. Siméon, "How may i serve you?: a robot companion approaching a seated person in a helping context," in Proceedings of the 1st ACM SIGCHI/SIGART conference on Humanrobot interaction. ACM, 2006, pp. 172-179.

[5] D. He, H. Ren, W. Hua, G. Pan, S. Li, and Z. Wu, "Flyingbuddy: augment human mobility and perceptibility," in Proceedings of the 13th international conference on Ubiquitous computing. ACM, 2011, pp. 615-616.

[6] J. Nagi, A. Giusti, G. A. Di Caro, and L. M. Gambardella, "Human control of uavs using face pose estimates and hand gestures," in Proceedings of the ACM/IEEE international conference on Humanrobot interaction, 2014, pp. 252-253.

[7] D. Helbing and P. Molnar, "Social force model for pedestrian dynamics," Physical review E, vol. 51, no. 5, p. 4282, 1995.

[8] G. Hinton, N. Srivastava, and K. Swersky, "Rmsprop: Divide the gradient by a running average of its recent magnitude," Neural networks for machine learning, Coursera lecture 6e, 2012.

[9] L. Davis, "Genetic algorithms and simulated annealing," 1987.

[10] G. Ferrer, A. Garrell, M. Villamizar, I. Huerta, and A. Sanfeliu, "Robot interactive learning through human assistance." 2013.

[11] E. T. Hall, "The hidden dimension," 1966.

[12] F. Zanlungo, T. Ikeda, and T. Kanda, "Social force model with explicit collision prediction," EPL (Europhysics Letters), vol. 93, no. 6, p. 68005, 2011.

[13] M. Luber, J. A. Stork, G. D. Tipaldi, and K. O. Arras, "People tracking with human motion predictions from social forces," pp. 464-469, 2010.

[14] L. Zhu, X. Cheng, and F.-G. Yuan, "A 3d collision avoidance strategy for uav with physical constraints," Measurement, vol. 77, pp. 40-49, 2016. 